\title{
蛠 \\ NeuroRehab News \\ El uso de los videojuegos en la prevención del deterioro cognitivo en personas mayores
}

Juego Terapéutico

\section{Resumen:}

다 ldeterioro cognitivo está relacionado, entre otros factores, con la pérdida de sustancia gris o cuEurpos neuronales en un área del cerebro llamada hipocampo, dándose de forma más prevalente en personas mayores. Por tanto, deben establecerse herramientas de tratamiento adecuadas para intentar demorar y minimizar dicha atrofia a nivel cerebral con el fin de preservar una correcta salud cognitiva. Los videojuegos surgen como una posible opción ante esta alteración debido a que los resultados sugieren, no solo que mejora funciones cognitivas como la memoria, si no también otras relacionadas con el aprendizaje y planificación de tareas motoras, el equilibrio o el control motor.

El hipocampo es una región del cerebro relacionada con funciones cognitivas, como la memoria. En el hipocampo se localiza la sustancia gris, formada por los cuerpos de las neuronas de esta área. La disminución de sustancia gris en el hipocampo es un elemento predictor para alteraciones neurológicas y deterioro cognitivo relacionado con enfermedades como el Alzheimer, afectando estos trastornos principalmente a personas mayores. Recientes estudios postulan que el entrenamiento cognitivo específico de determinadas tareas permite aumentar la materia gris en esta región cerebral, tratando así de prevenir el deterioro cognitivo.

En 2017 Greg L. West y Benjamin Rich Zendel, dos investigadores que desarrollan sus estudios en el campo de la neuropsicología y cognición en la universidad canadiense de Montreal, junto con sus colaboradores propusieron los videojuegos de plataforma 3D para llevar a cabo dicho entrenamiento, ya que precisan de memoria espacial, aprendizaje, planificación y coordinación motora. Postularon que no solo aumentaría la sustancia gris del hipocampo, si no que podría beneficiar a otras regiones como el cerebelo, implicado en la coordinación del movimiento; o la corteza prefrontal dorsolateral, relacionada con tareas como memoria, atención y planificación de acciones (West et al., 2017).

Iniciaron un estudio con 48 participantes adultos mayores, sin experiencia previa en el uso de videojuegos. Previa y posteriormente a la intervención fueron sometidos a pruebas de evaluación cognitiva general, memoria a corto plazo y una resonancia magnética para valorar los cambios en la materia gris. Los participantes fueron asignados aleatoriamente en tres grupos, recibiendo un entrenamiento diferente en cada uno de ellos.

El primer grupo realizó un entrenamiento empleando el videojuego de plataforma tridimensional Super Mario 64, en el que debían explorar un entorno virtual e ir superando diferentes niveles. Otro grupo empleó un programa informático y un piano para reproducir canciones y superar lecciones. El tercer grupo únicamente realizó las pruebas de evaluación, pero no llevó a cabo ninguna actividad específica durante el estudio. Las actividades fueron practicadas en el domicilio durante 6 meses, en los que debían realizar al menos 5 días a la semana 30 minutos de la actividad asignada. Puesto que no tenían experiencia previa, tras la instalación del material recibieron algunas instrucciones por parte del grupo de investigación para desenvolverse en la actividad correctamente.

Tras el entrenamiento, que conllevó finalmente unas 70 - 80 horas, se observó mejoría en cognición general únicamente en los participantes que emplearon videojuegos. En cuanto a la sustancia gris del hipocampo, aumentó de nuevo en las personas
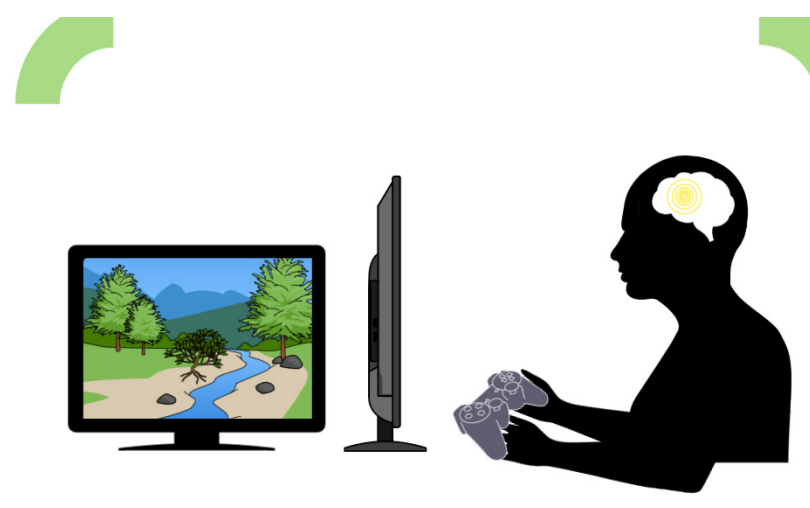

"Los juegos de plataforma tri-
dimensional promueven los
procesos de memoria espacial
y estrategias de navegación para crear un mapa cognitivo" 
que utilizaron videojuegos, simplemente se preservó en los que realizaron actividades musicales, mientras que aquellos que no realizaron ninguna actividad específica llegaron incluso a mostrar una disminución.

Además, en relación con otras áreas cerebrales, se hallaron incrementos de sustancia gris en la corteza prefrontal dorsolateral únicamente tras las actividades musicales y en el cerebelo en los grupos que emplearon tanto videojuegos como piano. Esto puede significar que la ejecución de actividades motoras en los videojuegos podría incluso mejorar la planificación y razonamiento, así como el equilibrio y la marcha en personas mayores.

Portanto, tras estudiar el impacto de los videojuegos en el hipocampo y en la memoria en adultos mayores, se puede establecer una relación positiva con el uso de nuevas actividades cognitivas, no solo para prevenir la atrofia de la materia gris del hipocampo debida a la edad, si no para aumentarla, tal y como indicaron estudios previos, tanto en personas mayores (Lövdén et al., 2012) como en adultos jóvenes (Kühn, Gleich, Lorenz, Lindenberger, \& Gallinat, 2014).

En cuanto a la explicación de estos resultados favorables en el incremento de sustancia gris, podría ser que los videojuegos impulsen el aprendizaje espacial, siendo esta una tarea dependiente del hipocampo, ya que es necesario recabar información del entorno virtual, por ejemplo, tomar árboles o edificios del juego como referencia, para poder establecer estrategias de navegación,
"Los videojuegos permiten mantener una buena salud cognitiva, evitando la disminución de materia gris y deterioro en el hipocampo"

alcanzar ubicaciones y desarrollar tareas con el fin de superar niveles. Esto sucede gracias al concepto de neuroplasticidad, la capacidad que presenta el cerebro para reorganizarse ante los diferentes estímulos del entorno, pudiendo ser esta modificación adaptativa o desadaptativa, en función de si ocurre para mejorar o deteriorar la función respectivamente.

Sin embargo, la utilización de este tipo de juegos puede verse limitado en personas mayores por problemas relacionados con el aprendizaje y coordinación necesarios para su uso, al no estar familiarizados con estas herramientas, llegando incluso a provocar abandono del entrenamiento. Podría ser interesante desarrollar alternativas cuya implantación sea más alcanzable en el caso de las personas de mayor edad, favoreciendo así un envejecimiento más saludable.

\section{Conclusión:}

En conclusión, los videojuegos se presentan como una herramienta útil en la prevención de trastornos neurodegenerativos en personas mayores. Aun así, son necesarias investigaciones en el futuro para descubrir qué aspectos específicos del entrenamiento con videojuegos son los causantes de los resultados positivos obtenidos. Además, dado que este tipo de alteraciones afectan principalmente a la población de mayor edad, sería interesante desarrollar herramientas de entrenamiento adaptadas a este grupo etario, con el fin de que puedan beneficiarse de los efectos positivos del entrenamiento cognitivo de una forma fácil, cómoda y accesible para esta población.

\section{Sobre este artículo:}

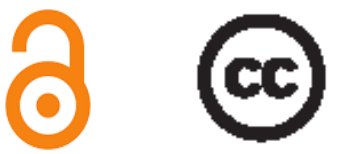

Fuente /s:

-Kühn, S., Gleich, T., Lorenz, R. C., Lindenberger, U., \& Gallinat, J. (2014). Playing Super Mario induces structural brain plasticity: gray matter changes resulting from training with a commercial video game. Molecular Psychiatry, 19;(2):265-271. https://doi. org/10.1038/mp.2013.120

-Lövdén, M., Schaefer, S., Noack, H., Bodammer, N. C., Kühn, S., Heinze, H. J., ... Lindenberger, U. (2012). Spatial navigation training protects the hippocampus against age-related changes during early and late adulthood. Neurobiology of Aging, 33;(3):620.e9-620.e22. https://doi.org/10.1016/j.neurobiolaging.2011.02.013

-West, G. L., Zendel, B. R., Konishi, K., Benady-Chorney, J., Bohbot, V. D., Peretz, I., \& Belleville, S. (2017). Playing Super Mario 64 increases hippocampal grey matter in older adults. PloS One, 12;(12):e0187779.

Fuente de la Imagen: imagen de NeuroRehabnews.com con fines unicamente ilustrativos.

Para citar este artículo: Herranz-Gómez A. El uso de los videojuegos en la prevención del deterioro cognitivo en personas mayores. NeuroRehab News 2020 mar; 4(1):e0061

Edición: Ferran Cuenca Martínez y Roy La Touche

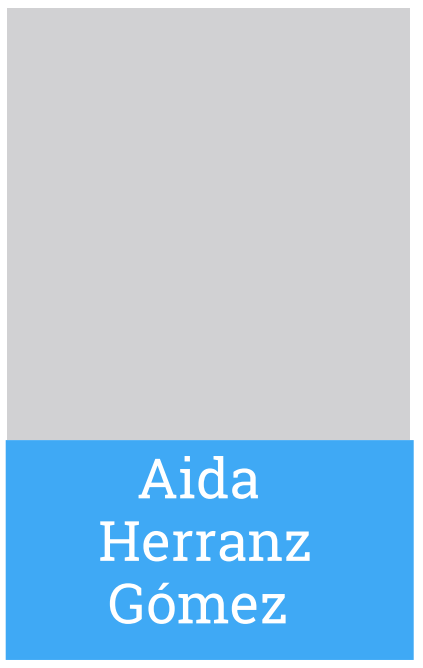

